\title{
Congenital Heart Disease in Maternal Phenylketonuria: Report from the Maternal PKU Collaborative Study
}

\author{
HARVEY L. LEVY, PER GULDBERG, FLEMMING GÜTTLER, WILLIAM B. HANLEY, \\ REUBEN MATALON, BOBBYE M. ROUSE, FRIEDRICH TREFZ, COLLEEN AZEN, \\ ELIZABETH N. ALLRED, FELIX DE LA CRUZ, AND RICHARD KOCH
}

\author{
Division of Genetics and Neuroepidemiology Unit, Children's Hospital and Departments of Pediatrics and \\ Neurology, Harvard Medical School, Boston, Massachusetts, U.S.A. [H.L.L., E.N.A.]; John F. Kennedy \\ Institute, Glostrup, Denmark [P.G., F.G.]; Hospital for Sick Children, Toronto, Ontario, Canada \\ [W.B.H.]; University of Texas Medical Branch at Galveston, Galveston, Texas, U.S.A. [R.M., B.M.R.]; \\ Children's Hospital of Reutlingen, Germany [F.T.]; Children's Hospital of Los Angeles, Los Angeles, \\ California, U.S.A. [C.A., R.K.]; and the National Institute of Child Health and Human Development,
} Bethesda, Maryland, U.S.A. [F. de la C.]

\section{ABSTRACT}

The frequency and types of congenital heart disease in offspring from pregnancies in women with hyperphenylalaninemia were examined in the international prospective Maternal Phenylketonuria Collaborative Study. Relationships of congenital heart disease in offspring to the basal blood phenylalanine level in the mother, metabolic control through diet during pregnancy, and phenylalanine hydroxylase mutations in mother and offspring were determined. The 416 offspring from 412 maternal phenylketonuria pregnancies that produced live births and 100 offspring from the 99 control pregnancies were included in this examination. Thirty-four of the 235 offspring (14\%; 95\% CI, 10.2 to $19.6 \%$ ) from pregnancies in phenylketonuric women with a basal phenylalanine level $\geq 900 \mu \mathrm{M}(15 \mathrm{mg} / \mathrm{dL})$ [normal blood phenylalanine $<120 \mu \mathrm{M}(2 \mathrm{mg} / \mathrm{dL})]$ and not in metabolic control [phenylalanine level $\leq 600 \mu \mathrm{M}(10 \mathrm{mg} / \mathrm{dL})$ ] by the eighth gestational week had congenital heart disease compared with one control offspring ( $1 \%)$ with congenital heart disease. One offspring among the $50(2 \%)$ from mothers with nonphenylketonuria mild hyperphenylalaninemia also had congenital heart disease. Coarctation of the aorta and hypoplastic left heart syndrome were overrepresented compared with expected percentages among those with congenital heart disease in the general population. A basal maternal phenylalanine level $>1800$ $\mu \mathrm{M}(30 \mathrm{mg} / \mathrm{dL})$ significantly increased the risk for bearing a child with congenital heart disease $(p=0.003)$. Phenylalanine hydroxylase mutations in the mothers and offspring did not have an independent relationship to congenital heart disease but were related through the basal maternal phenylalanine levels. The data in this study indicate that a basal maternal phenylalanine level of $900 \mu \mathrm{M}$ may be a threshold for congenital heart disease, that women with the most severe degree of phenylketonuria are at highest risk for bearing such a child, and that prevention of the congenital heart disease requires initiation of the low phenylalanine diet before conception or early in pregnancy with metabolic control no later than the eighth gestational week. (Pediatr Res 49: 636-642, 2001)

PKU, phenylketonuria

CHD, congenital heart disease

PAH, phenylalanine hydroxylase

MPKUCS, Maternal Phenylketonuria Collaborative Study

APL, assigned blood phenylalanine level

MHP, mild hyperphenylalaninemia
Maternal PKU is one of the most teratogenic of pregnancy complications (1). When these pregnancies are untreated,

Received April 5, 2000; accepted December 12, 2000.

Correspondence and reprint requests: Harvey L. Levy, M.D., Children's Hospital, 300 Longwood Ave., IC-106, Boston, MA 02115, U.S.A.; e-mail: harvey.levy@ tch.harvard.edu

Supported by contract NO1-HD-2-3149 from the National Institute of Child Health and Human Development, (National Institutes of Health), by grant MO1-RR02172 from the National Center for Research Resources (National Institutes of Health), National Health Research and Development Program Project 6606-3265 from Health and Welfare Canada, Danish Medical Research Council, EC Programme BIOMED I (Area 3: Human Genome Analysis), and the Danish Research Academy.
$>90 \%$ of the offspring suffer microcephaly and mental retardation. The frequencies of intrauterine growth retardation and CHD among offspring from these pregnancies are also much higher than expected in the general population, the former $\geq 40 \%$ and the latter $\geq 12 \%$ (2).

The problem of CHD is particularly interesting. Although its frequency among offspring from untreated maternal PKU pregnancies is increased 15 -fold above the general population frequency of $0.8 \%(3)$, it is still far less frequent than microcephaly and mental retardation among offspring from these 
pregnancies. Thus, cardiogenesis seems to be undisturbed in $85 \%$ or more of untreated maternal PKU pregnancies. Second, there is evidence that some women with PKU may be at particularly high risk for bearing children with CHD, but even in these families only some of the offspring have had this complication (4). Third, certain cardiac anomalies, especially tetralogy of Fallot and coarctation of the aorta, have been inordinately frequent among the types of CHD reported in these offspring (4). Finally, the heart is the only major fetal organ other than the brain affected by maternal PKU (5).

Although it seems clear that the occurrences of both brain and cardiac complications in the fetus are related to the degree of hyperphenylalaninemia in the pregnant woman, there being no excess of CHD and lower frequencies of microcephaly and mental retardation when the maternal blood phenylalanine level is $<600 \mu \mathrm{M}$ (2) (to convert the phenylalanine concentration from $\mu \mathrm{M}$ to $\mathrm{mg} / \mathrm{dL}$, divide by 60 ), the absence of CHD in the majority of untreated maternal PKU pregnancies suggests that aberrant cardiogenesis requires more than only the factor(s) that produces the brain effect. This additional requirement might be an unusually high phenylalanine level or an interaction between the high phenylalanine level and a second factor.

In considering agents that could interrelate with hyperphenylalaninemia in producing $\mathrm{CHD}$, the mutation(s) in the PAH gene is a potential candidate. This gene encodes $\mathrm{PAH}$, the liver enzyme defective in PKU. More than 400 such mutations have been identified (6). Most individuals with PKU are doubly heterozygous, having two different mutant alleles at the PAH locus (7), and only one of these two mutations can be inherited by the fetus from a phenylketonuric parent. Although the PAH genotype is highly correlated with the degree of hyperphenylalaninemia $(8,9)$, some maternal PAH genotypes might confer a particular risk of CHD. Similarly, the PAH mutation in the offspring might influence the occurrence and type of CHD.

In the international MPKUCS, we have had an opportunity to investigate these possibilities by comparing the degree of maternal hyperphenylalaninemia and the PAH mutations in enrolled women and their offspring with the occurrence and types of CHD in these offspring.

\section{METHODS}

Subjects. The MPKUCS is a prospective study that began in 1984 to investigate the effectiveness of a phenylalaninerestricted diet in reducing the teratogenic effects of maternal PKU (10). Participants include the major PKU treatment centers in the United States and Canada, and several centers in Germany (11). During the pregnancy study phase of the MPKUCS, which ended in May 1995, all hyperphenylalaninemic women known or reported to the centers who were planning a pregnancy or were already pregnant were requested to enroll in the study. Control pregnancies in normal women known to the participating centers were also enrolled (12) The study protocol has been described (11). Specific areas of the protocol relevant to the results we are describing include determination of the biochemical phenotype on the basis of a basal phenylalanine level, or APL, selected as the highest of two or three plasma phenylalanine levels on an unrestricted diet measured by amino acid analyzer or the fluorometric method (13) when the subject was not pregnant, dietary treatment with measurements of blood phenylalanine levels during the maternal PKU pregnancies, PAH genotypes in the mother and the PAH mutation in the offspring, fetal ultrasonography during each trimester (14), and medical examination of the offspring at birth, $6 \mathrm{mo}$, and annually thereafter through childhood. Informed consent was required, and the study was approved by the institutional review boards of all participating centers.

The study enrolled 572 pregnancies in women with PKU or non-PKU MHP. Of these, 79 were terminated, 75 spontaneously aborted, three were ectopic pregnancies, three were stillbirths, and 412 resulted in live births, producing 416 offspring (including four sets of twins). An additional 99 normal pregnancies were enrolled as controls, resulting in 100 live-born offspring. Treatment with a phenylalanine-restricted diet was initiated before conception or as early as possible after conception in women with blood phenylalanine levels $>600$ $\mu \mathrm{M}$ (or, on occasion, $>360 \mu \mathrm{M}$ ). Metabolic control was defined as a blood phenylalanine level $\leq 600 \mu \mathrm{M}$.

PAH genotyping. The maternal PAH genotypes were determined in blood specimens obtained by venipuncture as described $(7,15)$. For determination of the maternally transmitted PAH mutations in the offspring, dried blood filter paper specimens or postmortem specimens embedded in paraffin were obtained and examined for PAH mutations. The Chelex-100 method was used for DNA extraction from the dried blood specimens $(16,17)$, and the QIAamp Tissue Kit (Quigen Inc, Santa Clarita, CA, U.S.A.) was used for extraction of DNA from paraffin-embedded tissue. The relevant exonic regions of the PAH gene were PCR-amplified from both maternal and offspring DNA, and the amplification products were analyzed by denaturing gradient gel electrophoresis. Identity of the offspring mutation was based on the band pattern.

Statistical methods. Ninety-five percent binomial CIs were calculated for percentages. The relationship of increasing maternal APL to the risk of having a child with CHD was evaluated with a nonparametric test for trend (18). The relationship between PAH mutations and offspring with or without CHD was evaluated with Fisher's exact test.

\section{RESULTS}

Congenital heart disease. CHD was identified and confirmed by postnatal echocardiography or autopsy in 34 offspring from pregnancies in women with PKU and in one offspring from a mother with MHP. These include 33 live-born infants and two fetuses from pregnancies terminated in the second trimester because of CHD considered inoperable and incompatible with postnatal survival. The 33 phenylketonuric mothers of these offspring had APL $\geq 900 \mu \mathrm{M}$ and did not achieve metabolic control before the eighth gestational week. The 34 affected offspring from these maternal PKU pregnancies represent $14 \%$ (95\% CI, 10.2 to $19.6 \%)$ of the 235 offspring from mothers in this category of maternal PKU. Only one among the100 offspring (1\%) from the normal control 
Table 1. Cardiac defects and their percentages in offspring with congenital heart disease in the MPKUCS

\begin{tabular}{|c|c|c|c|}
\hline \multirow[b]{2}{*}{ Defect } & \multicolumn{2}{|c|}{$\begin{array}{l}\text { Number and percentages } \\
\text { among offspring* }\end{array}$} & \multirow{2}{*}{$\begin{array}{l}\text { Percentages among all children } \\
\text { with congenital heart disease } \dagger \\
\%(95 \% \mathrm{CI})\end{array}$} \\
\hline & $n$ & $\%(95 \% \mathrm{CI})$ & \\
\hline Tetralogy of Fallot & 6 & $17(7-34)$ & $9 \quad(8.7-9.6)$ \\
\hline Patent ductus arteriosus & 5 & $14(5-30)$ & $6 \quad(5.2-6.0)$ \\
\hline Hypoplastic left heart syndrome & 4 & $11(3-27)$ & $2(1.7-2.1)$ \\
\hline Pulmonic stenosis & 2 & $6(1-19)$ & $10 \quad(9.3-10.3)$ \\
\hline Atrial septal defect & 1 & $3-$ & $6(5.4-6.2)$ \\
\hline Mitral insufficiency with cleft mitral valve & 1 & $3-$ & $2(1.7-2.2)$ \\
\hline Mitral atresia & 1 & $3-$ & $2(1.7-2.2)$ \\
\hline Bicuspid aortic valve with aortic insufficiency & 1 & $3-$ & $<1(0.36-0.58)$ \\
\hline
\end{tabular}

* Number and percentage of offspring with the lesion among the 35 offspring with CHD in the MPKUCS.

$\dagger$ From Fyler DC 1992 Trends. In: Fyler DC (ed) Nadas’ Pediatric Cardiology. Mosby-Year Book, St. Louis, pp 273-280. There were 15,331 infants included in this compilation.

$\ddagger$ Only those offspring with patent ductus arteriosus requiring surgical closure or considered medically significant are included.

pregnancies had $\mathrm{CHD}$, and the frequency of $\mathrm{CHD}$ in the general population is $0.8 \%$ ( $95 \% \mathrm{CI}, 0.74$ to $0.89 \%$ ) (3). There was no CHD among the 131 offspring from maternal PKU pregnancies in which the maternal APL was $<900 \mu \mathrm{M}$ or in which metabolic control was achieved before the eighth gestational week. The mother with MHP who bore a child with CHD had an APL of $516 \mu \mathrm{M}$ and received no treatment during the pregnancy. Her offspring represents $2 \%$ of the 50 offspring from mothers with untreated MHP.

The types of cardiac defects identified in the affected offspring are listed in Table 1. The most frequent was coarctation of the aorta in seven offspring $(20 \%)$, followed by tetralogy of Fallot in six (17\%). Other frequent defects included patent ductus arteriosus (14\%), hypoplastic left heart syndrome $(11 \%)$, and ventricular septal defect (11\%). Both coarctation of the aorta and hypoplastic left heart syndrome were overrepresented in offspring with CHD compared with the percentages of these defects among children with CHD in the general population, with no overlapping of the $95 \%$ CIs. Tetralogy of Fallot and patent ductus arteriosus each occurred approximately twice as frequently among the affected offspring as in the general population of CHD but with CIs that overlapped those of the general population. Conversely, the percentage of ventricular septal defect in affected offspring was low compared with the general population of CHD. The single control offspring with CHD had a ventricular septal defect.

Maternal APL in occurrence and severity of CHD. Could the degree of hyperphenylalaninemia in the mother correlate with the risk of producing an offspring with $\mathrm{CHD}$ and the severity of the lesion? Table 2 examines the frequency of bearing a child with CHD in relation to the APL among phenylketonuric mothers with APL $\geq 900 \mu \mathrm{M}$ and not in metabolic control by the eighth gestational week. Among these at-risk mothers, $>50 \%$ of those bearing an offspring with CHD had an APL $>1800 \mu \mathrm{M}$, representing 33\% of the mothers in this APL category $(p=0.003)$. Thus, mothers with an APL $>$ $1800 \mu \mathrm{M}$ were significantly overrepresented among those who bore children with CHD. From these data (Table 2), it appears that having an APL $>1800 \mu \mathrm{M}$ and not being in metabolic control by the eighth gestational week confers a risk of $1: 3$ for having a child with CHD. Even a lower APL of 1501-1800 $\mu \mathrm{M}$ confers a risk of almost 1:5 for CHD unless metabolic control is attained by the eighth gestational week. However, the severity of CHD was not related to the degree of maternal APL. For instance, the range of APL in mothers bearing children with very severe and inoperable lesions that resulted in demise of the infant was $1518-2124 \mu \mathrm{M}$, whereas the range among mothers bearing children with coarctation of the aorta and patent ductus arteriosus was 2070-2604 $\mu \mathrm{M}$.

PAH mutations. The maternal PAH genotype and offspring PAH mutation could be determined in 23 families with CHD. In an additional family, only one of the two mutant alleles in the mother was characterized. These mothers harbored $21 \mathrm{PAH}$ mutations. Table 3 lists the frequencies of PAH mutations in these women compared with frequencies in those with maternal PKU who did not have children with CHD but who also had an APL $\geq 900 \mu \mathrm{M}$ and were not in metabolic control by the eighth gestational week. Only mutations occurring in more than one mother are listed. The most frequent mutation, R408W, was equally represented in affected and unaffected families. Three mutations (I65T, P281L, and F39L) were considerably but not significantly more frequent in mothers who had children with CHD. Table 3 also lists the mutations and their frequencies in offspring from both sets of mothers. Only I65T was significantly more frequent in offspring with CHD.

Table 2. Frequencies of at-risk mothers with treated maternal PKU* who bore offspring with CHD, categorized by APL

\begin{tabular}{ccccc}
\hline & \multicolumn{4}{c}{ APL $(\mu \mathrm{M})$} \\
\cline { 2 - 5 } Mothers & $900-1200$ & $1201-1500$ & $1501-1800$ & $1801-3000$ \\
\hline Total number & 30 & 46 & 49 & 51 \\
$\%$ with CHD & $13 \%(4)$ & $7 \%(3)$ & $18 \%(9)$ & $33 \%(17) \dagger$ \\
offspring $(n)$ & & & & \\
\hline
\end{tabular}

* APL $\geq 900 \mu \mathrm{M}$ and not in metabolic control by the eighth gestational week.

$\dagger p=0.003$ 
Table 3. Percentages of mothers with and without children with CHD and of affected offspring according to PAH gene mutations

\begin{tabular}{|c|c|c|c|c|c|c|}
\hline \multirow[b]{3}{*}{ Mutation* } & \multicolumn{2}{|c|}{ Mothers $\dagger$} & \multirow[b]{3}{*}{$p$} & \multicolumn{2}{|c|}{ Offspring } & \multirow[b]{3}{*}{$p$} \\
\hline & $\begin{array}{l}\text { Offspring with } \\
\text { CHD }(n=24)\end{array}$ & $\begin{array}{l}\text { Offspring without } \\
\text { CHD }(n=51)\end{array}$ & & $\begin{array}{l}\text { with CHD } \\
(n=26)\end{array}$ & $\begin{array}{l}\text { without CHD } \$ \\
\qquad(n=72)\end{array}$ & \\
\hline & & & & \multicolumn{2}{|c|}{$\%(95 \% \mathrm{CI})$} & \\
\hline R408W & $46(26-67)$ & $41(28-56)$ & $\frac{P}{0.45}$ & $27(12-48)$ & $28(18-40)$ & 0.58 \\
\hline IVS12nt1 & $17(5-37)$ & $25(14-40)$ & 0.30 & $4(0.1-20)$ & $11(5-21)$ & 0.26 \\
\hline $\mathrm{I} 65 \mathrm{~T}$ & $17(5-37)$ & $6(1-16)$ & 0.14 & $15(4-35)$ & 0 & 0.004 \\
\hline F39L & $13(3-32)$ & $4(0.5-13)$ & 0.18 & $4(0.1-20)$ & $3(0.3-10)$ & 0.61 \\
\hline $\mathrm{R} 243 \mathrm{X}$ & $8(1-27)$ & $2(0.1-10)$ & 0.24 & 0 & 0 & - \\
\hline R158Q & $4(0.1-21)$ & $4(0.5-13)$ & 0.69 & $4(0.1-20)$ & $3(0.3-10)$ & 0.61 \\
\hline Y414C & $4(0.1-21)$ & $8(2-19)$ & 0.48 & $4(0.1-20)$ & $6(2-14)$ & 0.60 \\
\hline
\end{tabular}

* Mutations occurring in only one maternal PAH allele are not listed. Because each mother harbors two mutant alleles, she may appear twice in the list of maternal mutations.

$\dagger$ Only mothers with APL $\geq 900 \mu \mathrm{M}$ and not in metabolic control by eighth gestational week are included.

$\$$ In families with multiple unaffected offspring, one offspring was chosen at random (coin flip) to represent the family to avoid overrepresentation of certain mutations in offspring.

In three families the mothers bearing offspring with CHD have also had offspring without CHD. The complete genotypes in these families are listed in Table 4. In one family the two offspring with CHD inherited the I65T mutation. (The pregnancies resulting in these two offspring occurred before enrollment in the MPKUCS began.) However, in the second family two of the three offspring who do not have CHD carry the I65T mutation. Moreover, in this family and in the third family, R408W occurred in both affected and unaffected offspring. In these three families, therefore, there was no relationship between CHD in the offspring and inheritance of a specific PAH mutation, indicating that a relationship associated with I65T in the offspring suggested by Table 3 is likely caused by chance.

As implied from Table 2, CHD is most likely when the mother has a severe degree of PKU. Nevertheless, mothers with less than classic PKU may still have pregnancies complicated by CHD. Table 5 lists the PAH genotype and presumed biochemical phenotype determined according to Guldberg et al. (9), as well as the APL in the 23 mothers completely genotyped who had an offspring with CHD. Most had a genotype consistent with classic PKU, but eight could be classified as having moderate PKU and two as having mild PKU. However, four of the eight with a moderate PKU genotype and one of the two mothers classified as having only mild

Table 4. PAH mutations in families with and without offspring affected by CHD

\begin{tabular}{|c|c|c|c|}
\hline \multirow[b]{2}{*}{ Family } & \multirow[b]{2}{*}{ Maternal genotype } & \multicolumn{2}{|c|}{ Offspring mutation } \\
\hline & & With CHD & Without CHD \\
\hline \multirow[t]{2}{*}{1} & IVS12nt1/I65T & $\mathrm{I} 65 \mathrm{~T}$ & IVS12nt1 \\
\hline & & $\mathrm{I} 65 \mathrm{~T}$ & \\
\hline \multirow[t]{3}{*}{2} & $\mathrm{R} 408 \mathrm{~W} / \mathrm{I} 65 \mathrm{~T}$ & R408W & R408W \\
\hline & & & $\mathrm{I} 65 \mathrm{~T}^{*}$ \\
\hline & & & I65T* \\
\hline \multirow[t]{2}{*}{3} & $\mathrm{R} 408 \mathrm{~W} / \mathrm{P} 281 \mathrm{~L}$ & R408W & R408W \\
\hline & & & P281L \\
\hline
\end{tabular}

* These two offspring were from pregnancies that occurred before enrollment in the MPKUCS began, thus they are not included in study data.
PKU had APLs that were in the range of the mothers with classic PKU. This inconsistency of biochemical phenotype when an individual harbors a mutation with residual PAH activity, as do those with moderate and mild PKU, has been well documented $(8,9)$. These data emphasize the priority of the degree of hyperphenylalaninemia in the occurrence of CHD in maternal PKU.

There was also no relationship between specific PAH mutations and the type of CHD. Eight mutations were present in mothers bearing children with coarctation of the aorta. Similarly, six mutations were represented in mothers bearing children with the hypoplastic left heart syndrome, six in mothers of children with ventricular septal defect, five in mothers bearing children with tetralogy of Fallot, and five in mothers having children with patent ductus arteriosus. The mutations in the offspring were similarly spread across the spectrum of PAH mutations found in the study.

Fetal ultrasonography. Fetal ultrasonography was performed in 30 of the 34 pregnancies that resulted in offspring with CHD. The presence of a cardiac anomaly was identified at 19-22 wk gestation in seven of these pregnancies. In three additional fetuses, the cardiac lesions were identified by ultrasonography at 34,36 , and 38 wk gestation, after what were considered to be normal midtrimester examinations. Among the remaining 20 offspring who were examined by fetal ultrasonography, the five with isolated patent ductus arteriosus could not have been identified prenatally and the remaining 15 were also considered normal. Thus, most of the cardiac lesions potentially identifiable by midtrimester ultrasonography were not identified. The lesions identified were similar to those not identified, including tetralogy of Fallot, coarctation of the aorta, and hypoplastic left heart syndrome.

\section{DISCUSSION}

In this prospective study of maternal hyperphenylalaninemia, all but one instance of CHD occurred when the APL in the mother was $\geq 900 \mu \mathrm{M}$ and the pregnancy was not in metabolic control by the eighth week of gestation. Among all such treated 
Table 5. Maternal PAH genotypes when offspring has CHD

\begin{tabular}{lrcc}
\hline \multicolumn{2}{c}{ PAH mutation genotype } & $\begin{array}{c}\text { Biochemical phenotype } \\
\text { predicted on basis of } \\
\text { PAH genotype }\end{array}$ & $\begin{array}{c}\text { APL } \\
(\mu \mathrm{M})\end{array}$ \\
\hline 1043del11bp & R408W & ClassicPKU & 2600 \\
R408W & R243X & ClassicPKU & 2370 \\
R408W & P281L & ClassicPKU & 2120 \\
P281L & S16fsdelCT & ClassicPKU & 2080 \\
R408W & IVS12nt-1 & ClassicPKU & 1950 \\
R158Q* & F299C & ClassicPKU & 1950 \\
R408W & R242Q & ClassicPKU & 1850 \\
P281L & R408W & ClassicPKU & 1840 \\
R408W & R408W & ClassicPKU & 1820 \\
R408W & R408W & ClassicPKU & 1800 \\
S349P & R252W & ClassicPKU & 1790 \\
IVS12nt-1 & R243X & ClassicPKU & 1700 \\
E280K & E280K & ClassicPKU & 1500 \\
I65T* & IVS12nt-1 & ModeratePKU & 1880 \\
I65T* & IVS8nt-1 & ModeratePKU & 1700 \\
F39L* & T81P & ModeratePKU & 1580 \\
F39L* & E280K & ModeratePKU & 1570 \\
R261Q* & IVS12nt-1 & ModeratePKU & 1480 \\
I65T* & R408W & ModeratePKU & 1460 \\
F39L* & D84Y & ModeratePKU & 1420 \\
I65T* & E280K & ModeratePKU & 1180 \\
L48S* & R408W & MildPKU & 1920 \\
Y414C* & R408W & MildPKU & 1015 \\
\hline
\end{tabular}

* Mutations associated with residual PAH activity in vitro or apparent residual activity in vivo $(8,9)$.

$\dagger$ According to classification of Guldberg et al. (9).

maternal PKU pregnancies in the study, $14 \%$ of the offspring had CHD. This percentage is essentially the same as the 12 to $15 \%$ frequency of CHD among offspring from untreated maternal PKU pregnancies with maternal blood phenylalanine level $\geq 1000 \mu \mathrm{M}$ reported in an international survey (2) and far exceeds the frequencies of $0.8 \%$ for $\mathrm{CHD}$ in general populations $(3,19)$ and $1 \%$ among offspring from control pregnancies in this study. The single offspring with CHD when the maternal hyperphenylalaninemia was $<900 \mu \mathrm{M}$ in this study (2\%) may be compared with data from an international survey that included two instances of CHD among 77 untreated pregnancies $(2.6 \%)$ with maternal hyperphenylalaninemia $<$ $900 \mu \mathrm{M}(2)$.

Only 10 of the 35 offspring with CHD were identified prenatally by fetal ultrasonography. Thus, the careful follow-up of offspring in the MPKUCS was a critical feature of the study. This included annual examinations beyond the neonatal period, as almost $50 \%$ of children with CHD in the general population are not identified by the neonatal examination (19). Twenty-three of the affected offspring not detected prenatally in the MPKUCS were identified either on neonatal examination or by 6 mo of age. One of the remaining two offspring was not proven to have CHD until he was $4 \mathrm{y}$ old, despite having had a murmur noted since infancy. The other offspring was considered to have normal cardiac examinations until $8 \mathrm{y}$ of age, when he was found to have a bicuspid aortic valve and patent ductus arteriosis. This latter offspring is the only affected offspring whose mother has MHP rather than PKU.

It is still possible that CHD remains undetected in some offspring within the MPKUCS as echocardiography was not routinely performed except for only those offspring with findings considered by the attending physician to possibly indicate CHD. CHD not producing detectable murmurs or producing murmurs considered to be insignificant or CHD without functional impairment might remain undetected. Moreover, our definition of patent ductus arteriosus, i.e. requiring surgical closure or considered medically significant, might have excluded some affected offspring. Thus, the frequency of CHD found in the MPKUCS must be considered the minimal prevalence.

A major reason for fetal ultrasonography in the MPKUCS has been to detect congenital anomalies in time for the family to make informed decisions about continuing the pregnancy (1). This requires identification no later than midtrimester. Although this was often accomplished in one center (14), most infants with potentially identifiable CHD were either not identified by ultrasonography or not until the third trimester. This difference may reflect a particular interest in fetal ultrasonography for maternal PKU in a center as opposed to performance at multiple centers. Accordingly, whenever possible ultrasonography in maternal PKU pregnancies should be performed at a designated center with special interest and experience in identification of congenital anomalies, particularly CHD. Perhaps fetal echocardiography should also be part of prenatal evaluation in maternal PKU pregnancies. Nevertheless, because CHD was not identified in all affected offspring either by fetal ultrasonography or by physical examination during infancy, it seems reasonable to suggest that an echocardiogram be performed soon after birth on all maternal PKU offspring.

There is no doubt that maternal PKU increases the risk of bearing a child with CHD. Our study suggests that this risk is highest when the maternal APL is exceptionally high. More than $50 \%$ of the mothers with levels of blood phenylalanine $\geq$ $900 \mu \mathrm{M}$ and not in metabolic control by the eighth gestational week (at-risk pregnancies) who bore children with CHD had a blood phenylalanine level $\geq 1500 \mu \mathrm{M}$, and most of these had a level $>1800 \mu \mathrm{M}$. Thus, there seems to be a particularly high risk of bearing a child with CHD if the maternal blood phenylalanine level is $>1800 \mu \mathrm{M}$ and metabolic control on diet is delayed until after 8 wk gestation $(p=0.003)$.

Our study also indicates that there might be a maternal hyperphenylalaninemia threshold of $900 \mu \mathrm{M}$ for the teratogenic effect of CHD. Only one mother who bore an affected child had a lower APL. CHD in this child could represent background frequency as this was the only case among 50 offspring from mothers with MHP, a frequency of $2 \%$ compared with the frequency of $1 \%$ among control offspring in the study. If there is a phenylalanine threshold for CHD, there is no known explanation for it. However, there is an embryologic explanation for the apparent requirement of metabolic control by the eighth gestational week for the prevention of CHD. Cardiogenesis occurs during the fourth to 10th gestational weeks, with the fourth to eighth weeks the highly sensitive period for cardiac teratogenesis (20). Thus, control of hyperphenylalaninemia initiated after the eighth week should have no effect in preventing CHD, although it might be effective in preventing or ameliorating other teratogenic effects of maternal PKU (21). 
The mothers bearing children with CHD harbored $21 \mathrm{PAH}$ mutations. This multiplicity of mutations is representative of the MPKUCS (7). No specific maternal mutation and only one mutation in the offspring (I65T) exclusively associated with CHD. However, even the latter singular association does not indicate that a PAH mutation is an independent risk factor for CHD in maternal PKU, as evidenced by two families of multiple offspring in which the I65T mutation was transmitted only to the affected offspring in one family and only to the unaffected offspring in the other family. Moreover, in one of the families as well as a third family, the R408W mutation was transmitted to both affected and unaffected offspring. Thus, the effect of the PAH mutation in the CHD of maternal PKU is most likely mediated through the expressed hyperphenylalaninemia.

Considering that the risk for bearing a child with CHD increases with APL $\geq 1500 \mu \mathrm{M}$, it was unexpected to find that almost half of the mothers with CHD offspring carried PAH mutations that are associated with residual PAH activity and relatively mild phenotypes (mild PKU or moderate PKU). However, each of these mutations (F39L, L48S, I65T, R158Q, and R261Q) has been associated with a wide range of metabolic phenotypes in vivo, spanning the range from classic PKU to mild PKU $(8,9)$. It is likely that these mutations are associated with highly fluctuating phenylalanine levels with the higher level contributing to the development of CHD in maternal PKU.

Although 11 different types of congenital heart lesions occurred among affected offspring in this study, two (coarctation of the aorta and hypoplastic left heart syndrome) were represented at a significantly higher percentage than expected in children with CHD. Coarctation of the aorta is an extracardiac vascular anomaly, suggesting that this area of development might be particularly vulnerable to hyperphenylalaninemia. In this respect, it is of interest that cardiovascular lesions in fetuses from pregnancies in the PKU mouse model $\left(\mathrm{Pah}^{\mathrm{enu} 2}\right)$ are primarily vascular (22). Although this similarity between mouse and human maternal PKU does not explain the relatively high frequency of a vascular anomaly in maternal PKU, it does suggest that the mouse model might be appropriate for pathophysiologic studies of CHD in maternal PKU.

Possible genetic factors in the production of CHD have recently received considerable attention (23). Mutations introduced into several genes in mice have produced maldevelopment of the heart. A targeted mutation deleting the gene encoding the homeobox transcription factor Nkx2-5 produces nonlooping of the linear heart tube (24), resulting in early embryonic death because of hemodynamic insufficiency (25). Abnormal looping of the heart tube in the mouse also results from replacement of the first coding exon of the Handl gene that encodes the bHLH transcription factor (26). In the human, familial atrial septal defect and atrioventricular conduction abnormalities can be caused by mutations in the Nkx2-5 transcription factor gene (27), and deletions in the distal region of chromosome $8 \mathrm{p}$ are associated with a variety of congenital cardiac lesions (28). These cardiac lesions are not among those associated with CHD in maternal PKU. Nevertheless, these findings illustrate that in maternal PKU, polymorphisms in genes that regulate cardiac development could interact with severe hyperphenylalaninemia in the fetus to result in CHD. Specifically, these polymorphisms might be maladaptive with phenotypic expression occurring only in the presence of hyperphenylalaninemia, analogous to the concepts of maladaptation in phenotypic expression of histidinemia, Hartnup disorder, and other biochemical entities (29-31). Further studies should examine this possibility.

Acknowledgments. Many participating centers throughout the United States, Canada, and Germany have been instrumental in the collection and transmission of data on maternal PKU. The authors are grateful to the physicians, nutritionists, and others at these centers. This study would not have been possible without the work and dedication of the six coordinators of the contributing centers (Deborah Lobbregt, Barbara Goss, Elizabeth Wenz, Lois Castiglioni, Wanda Schoonheyt, and Sonja Cipcic-Schmidt).

\section{REFERENCES}

1. Levy HL, Ghavami M 1996 Maternal phenylketonuria: a metabolic teratogen. Teratology 53:176-184

2. Lenke RR, Levy HL 1980 Maternal phenylketonuria and hyperphenylalaninemia: an international survey of the outcome of untreated and treated pregnancies. $\mathrm{N}$ Engl J Med 303:1202-1208

3. Mitchell SC, Korones SB, Berendes HW 1971 Congenital heart disease in 56,109 births: incidence and natural history. Circulation 43:323-332

4. Pierpont MEM, Sletten LJ, Smith CF, Berry H, Berry SA, Fisch RO 1995 Congenital cardiac malformations in offspring of mothers with phenylketonuria and hyperphenylalaninemia. Intern Pediatr 10:242-249

5. Brenton DP 1990 Cardiac defects in the children of mothers with high concentrations of plasma phenylalanine. Br Heart J 63:143-144

6. Nowacki PM, Byck S, Prevost L, Scriver CR 1998 PAH Mutation Analysis Consortium Database: 1997. Prototype for relational locus-specific mutation databases. Nucleic Acids Res 26:220-225 (also Web site http://www.mcgill.ca/pahdb)

7. Guldberg P, Levy HL, Hanley WB, Koch R, Matalon R, Rouse BM, Trefz F, de la Cruz F, Henriksen KF, Güttler F 1996 Phenylalanine hydroxylase gene mutations in the United States: report from the Maternal PKU Collaborative Study. Am J Hum Genet 59:84-94

8. Kayaalp E, Treacy E, Waters PJ, Byck S, Nowacki P, Scriver CR 1997 Human phenylalanine hydroxylase mutations and hyperphenylalaninemia phenotypes: a metanalysis of genotype-phenotype correlations. Am J Hum Genet 61:1309-1317

9. Guldberg P, Rey F, Zschocke J, Romano V, Francois B, Michiels L, Ullrich K, Hoffman GF, Burgard P, Schmidt H, Meli C, Riva E, Dianzani I, Ponzone A, Rey J, Güttler F 1998 A European multicenter study of phenylalanine hydroxylase deficiency: classification of 105 mutations and a general system for genotype-based prediction of metabolic phenotype. Am J Hum Genet 63:71-79

10. Koch R, Hanley W, Levy H, Matalon R, Rouse B, de la Cruz F, Azen C, Friedman EG 1990 A preliminary report of the collaborative study of maternal phenylketonuria in the United States and Canada. J Inher Metab Dis 13:641-650

11. Friedman EG, Koch R, Azen C, Levy H, Hanley W, Matalon R, Rouse B, Trefz F, de la Cruz F 1996 The International Collaborative Study on maternal phenylketonuria: organization, study design and description of the sample. Eur J Pediatr 155(suppl 1):S158-S161

12. Koch R, Levy HL, Matalon R, Rouse B, Hanley WB, Trefz F, Azen C, Friedman EG, de la Cruz F, Güttler F, Acosta PB 1994 The international collaborative study of maternal phenylketonuria: status report 1994. Acta Paediatr Suppl 407:111-119

13. McCaman M, Robins E 1962 Fluorimetric method for the determination of phenylalanine in serum. J Lab Clin Med 59:885-890

14. Levy HL, Lobbregt D, Platt LD, Benacerraf BR 1996 Fetal ultrasonography in maternal PKU. Prenat Diagn 16:599-604

15. Guttler F, Azen C, Guldberg P, Romstad A, Hanley WB, Levy HL, Matalon R, Rouse BM, Trefz F, de la Cruz F, Koch R 1999 Relationship among genotype, biochemical phenotype, and cognitive performance in females with phenylalanine hydroxylase deficiency: report from the Maternal Phenylketonuria Collaborative Study. Pediatrics 104:258-262

16. Walsh PS, Metzger DA, Higuchi R 1991 Chelex 100 as a medium for simple extraction of DNA for PCR-based typing from forensic material. Biotechniques 10:506-513

17. Guttler F, Henriksen K, Guldberg P, Levy HL 1997 Confirmatory diagnosis of neonatal phenylalanine hydroxylase deficiency by mutation analysis of Guthrie Card DNA. In: Levy HL, Hermos R, Grady GF (eds) Proceedings of the Third International Meeting of the International Society for Neonatal Screening. IKON, Boston, pp $12-15$

18. Cuzick J 1985 A Wilcoxon-type test for trend. Stat Med 4:87-90

19. Ainsworth SB, Wyllie JP, Wren C 1999 Prevalence and clinical significance of cardiac murmurs in neonates. Arch Dis Child Fetal Neonatal Ed 80:F43-F45 
20. Sadler TW 1985 Langman's Medical Embryology. Williams \& Wilkins, Baltimore

21. Waisbren S 1999 Developmental and neuropsychological outcome in children born to mothers with phenylketonuria. Ment Retard Dev Disabil Res Rev 5:125-131

22. McDonald JD, Dyer CA, Gailis L, Kirby ML 1997 Cardiovascular defects among the progeny of mouse phenylketonuria females. Pediatr Res 42:103-107

23. Olson EN, Srivastava D 1996 Molecular pathways controlling heart development. Science 272:671-676

24. Biben C, Harvey RP 1997 Homeodomain factor Nkx2-5 controls left/right asymmetric expression of bHLH gene eHand during murine heart development. Genes Dev 11:1357-1369

25. Harvey RP 1996 NK-2 homeobox genes and heart development. Dev Biol 178:203-216

26. Firulli AB, McFadden DG, Lin Q, Srivastava D, Olson EN 1998 Heart and extraembryonic mesodermal defects in mouse embryos lacking the bHLH transcription factor Hand1. Nat Genet 18:266-70
27. Schott JJ, Benson DW, Basson CT, Pease W, Silberbach GM, Moak JP, Maron BJ, Seidman CE, Seidman JG 1998 Congenital heart disease caused by mutations in the transcription factor NKX2-5. Science 281:108-111

28. Devriendt K, Matthijs G, Van Dael R, Gewillig M, Eyskens B, Hjalgrim H, Dolmer B, McGaughran J, Bröndum-Nielsen K, Marynen P, Fryns J-P, Vermeesch JR 1999 Delineation of the critical deletion region for congenital heart defects, on chromosome 8p23.1. Am J Hum Genet 64:1119-1126

29. Scriver CR, Levy HL 1983 Histidinaemia. Part I: reconciling retrospective and prospective findings. J Inherit Metab Dis 6:51-53

30. Scriver CR, Mahon B, Levy HL, Clow CL, Reade TM, Kronick J, Lemieux B, Laberge C 1987 The Hartnup phenotype: Mendelian transport disorder, multifactorial disease. Am J Hum Genet 40:401-412

31. Scriver CR 1988 Nutrient-gene interactions: the gene is not the disease and vice versa Am J Clin Nutr 48:1505-1509 